\title{
REMESAS Y DESARROLLO EN AMÉRICA LATINA. UNA RELACIÓN EN BUSCA DE TEORÍA
}

\author{
ALEJANDRO I. CANALES CERÓN*
}

RESUMEN: En los últimos años, diversos gobiernos nacionales así como organismos multilaterales de ayuda al desarrollo dedican una especial atención a las remesas, como instrumento que podría contribuir a la reducción de la pobreza y al desarrollo de los países de origen de la migración internacional. En este artículo, desde una perspectiva crítica, queremos aportar algunos elementos conceptuales y empíricos, que permitan un mejor entendimiento de los alcances y limitaciones de las remesas como promotoras de procesos de desarrollo en las sociedades de origen de la migración. Por un lado, desde la macroeconomía, proponemos un modelo de análisis de la relación Remesas-Desarrollo que permite entender y evaluar los distintos impactos de las remesas familiares en función del carácter y modalidad que ellas asuman, así como de su significado y comportamiento específico como categoría económica. El supuesto que está detrás de este modelo analítico, es muy simple. El impacto de las remesas depende directamente de cómo ellas se incorporan a la estructura económica de cada sociedad, esto es, de cuál es el carácter y lógica que define su comportamiento macroeconómico. Por otro lado, con base en un modelo econométrico, estimamos los determinantes del comportamiento macroeconómico de las remesas. Los resultados del modelo indican que la dinámica y comportamiento macroeconómico de las remesas no se corresponde con el de un fondo de ahorro o inversión, sino más bien al de un ingreso salarial, que bajo la forma de transferencia familiar externa, contribuye a compensar los efectos negativos que las crisis recurrentes y los ciclos recesivos de la economía mexicana provocan sobre las economías familiares.

PALABRAS ClaVE: Desarrollo, Migración, Remesas, Economía, Modelo, Inversión.

ABSTRACT: Several governments and International organizations have lately paid attention to remittances as a tool that could contribute to the reduction of poverty and the development of the countries of origin of International migration. In this article, from a critical point of view, we submit some concepts and facts that allow a better understanding of the real role of remittances in promoting such a development process. First of all, from a macroeconomic analysis, we elaborate a model of the relationship between remittances and development that allows to understand and to evaluate the impacts of household remittances depending on their character and modality, as also their significance and specific behaviour as an economic category. The base of this analytical model is quite simple: the impact of remittances directly depends of the way they are incorporated to the economic structure of each society. Secondly, starting from an econometric model, we identify the determinants of the macroeconomic behaviour of remittances. The results of this model show that the dynamics of remittances are not related to an investment or saving fund, but to a wage fund, that in this case adopts the form of an external household transference, and that contributes to solve the negative effects over household of the reiterative crisis and recessions of the Mexican economy.

KEYWORDS: Development, Migration, Remittances, Economy, Model, Investment.

* Profesor-investigador, Universidad de Guadalajara. Correo electrónico: acanales@cucea.udg.mx 


\section{INTRODUCCIÓN}

ctualmente, en América Latina las remesas sobrepasan los 60 mil mi-
llones de dólares anuales. Este flujo representa uno de los principales
rubros de transferencias corrientes en la Balanza de Pagos, superando
en muchos casos el ingreso derivado de los principales rubros de exportación y la inversión extranjera directa, a la vez que son muy superiores a los recursos derivados de la cooperación internacional para el desarrollo. Estos volúmenes que han alcanzado las remesas, hacen que ellas se vuelvan no sólo visibles a los ojos de la sociedad, sino que las convierten en un importante tema de debate social, político y académico, en función de sus aparentes potencialidades como posible fuente de financiamiento del desarrollo local y regional.

En este contexto, diversos organismos internacionales (FMI, BID-FOMIN, UNCTAD, Banco Mundial, entre otros) junto con gobiernos nacionales y locales, enfocan su atención en las remesas como instrumento preferencial que podría contribuir a la reducción de la pobreza y a promover el desarrollo de los países de origen de la migración internacional (Terry, 2006; Ratha, 2003; Banco Mundial, 2004; Acosta et al., 2006; Alleyne, Kirton y Figueroa, 2008). En contraposición a este discurso predominante, varios organismos de la sociedad civil y no pocos académicos cuestionan estos nuevos enfoques, aduciendo que en esencia las remesas son transferencias privadas entre particulares, que por lo mismo, no pueden sustituir la responsabilidad del Estado y la acción del mercado en la promoción del desarrollo económico y el bienestar de la población (García-Zamora, 2005; Cortina et al., 2004; Canales, 2007b; Martínez, 2003; Lozano, 2005; de Haas, 2007; CePAL; 2006).

Más allá de estar de acuerdo con una u otra posición en torno al papel de las remesas como posible motor del desarrollo, resulta interesante comprobar que en este debate tiende a predominar cierto sesgo empiricista, en donde resulta más importante demostrar (o refutar, según sea el caso) la validez empírica de una hipótesis, que desentrañar las bases teóricas y conceptuales que podrían explicar esa misma hipótesis en torno a la relación Remesas-Desarrollo.

En efecto, el debate se ha centrado preferentemente en torno a cómo las remesas podrían promover un proceso de desarrollo económico en las regiones de origen de la migración, dejando amplios vacíos en torno al por qué las remesas podrían tener tal impacto (o no tenerlo, según sea el caso). En este marco, uno de los vacíos que más llama la atención, es precisamente, la ausencia de un marco teórico desde el cual se elabore una definición conceptual de las remesas, así como de sus relaciones con los procesos de desarrollo. Ante esta ausencia de teorías, lo más común es que se adopte directamente la definición contable de remesas que se usa para medir este tipo de transferencias a través de la Balanza de Pagos. En el mejor de los casos, se señalan los sesgos de esta definición contable, pero sólo en términos de aquellas transferencias que no quedan debidamente registradas como remesas familiares (Carling, 2007; Reinke y Patterson, 2005). 
Considerando este vacío teórico presente en el debate sobre la relación Remesas-Desarrollo, en este artículo nos interesa aportar algunos elementos conceptuales que permitan un mejor entendimiento de los alcances y limitaciones de las remesas como promotoras de procesos de desarrollo en las sociedades de origen de la migración. Para ello, presentamos una aproximación en dos fases.

Por un lado, desde la macroeconomía, proponemos un modelo de análisis de la relación Remesas-Desarrollo que permite entender y evaluar los distintos impactos de las remesas familiares en función del carácter y modalidad que ellas asuman, así como de su significado y comportamiento específico como categoría económica. El supuesto que está detrás de este modelo analítico es muy simple. El impacto de las remesas depende directamente de cómo ellas se incorporan a la estructura económica de cada sociedad, esto es, de cuál es el carácter y lógica que define su comportamiento macroeconómico.

Por otro lado, presentamos un modelo econométrico a través del cual estimamos los determinantes macroeconómicos de las remesas. En particular, estos determinantes nos permiten demostrar empíricamente el carácter y significado macroeconómico de las remesas, y por tanto, señalar los ámbitos y limitaciones de sus posibles impactos.

\section{REMESAS Y DESARROLLO. UN PARADIGMA EN BUSCA DE TEORÍA}

Resulta interesante comprobar, que aunque no parece haber un consenso en torno al significado y magnitud de los efectos sociales e impactos económicos de las remesas, a nivel mundial, tiende a predominar una posición celebratoria en torno a sus posibles efectos en las economías perceptoras del Tercer Mundo.

$\mathrm{Al}$ respecto, en la literatura que sustenta esta tendencia oficialista, podemos identificar dos tipos de discursos y análisis.

I. Por un lado, encontramos el discurso y textos de organismos internacionales, que suelen centrar su argumentación en propuestas que, desde su perspectiva, permitirían un mejor aprovechamiento de las potencialidades de las remesas. Se trata en general, de textos sin mayor sustentación teórica ni empírica, que buscan más bien, imponer una visión en relación al debate en torno a las remesas.

II. Por otro lado, cada vez son más frecuentes artículos académicos que, con base en modelos econométricos, buscan dar sustento empírico a estos modelos de acción política derivados del discurso predominante en los organismos internacionales y no pocos gobiernos nacionales.

i) En el primer caso, diversos autores y organismos internacionales de ayuda al desarrollo (BID, FMI, OECD, Banco Mundial, UNCTAD, por citar algunos) argumentan que, dada la magnitud y tendencia de las remesas, ellas suelen representar uno 
de los principales rubros de transferencias corrientes en la Balanza de Pagos de muchos países en desarrollo o de reciente industrialización; constituyen una verdadera inyección de recursos económicos en sectores específicos de las economías regionales y locales (Terry, 2006; Orozco y Wilson, 2005; Hugo, 2005; Chami et al., 2003; FOMin, 2004).

En particular, este discurso enaltece el papel de las remesas desde al menos dos ámbitos o dimensiones, a saber:

- Por su papel como promotoras del desarrollo económico, tanto directamente a través del financiamiento de proyectos productivos e infraestructura social, como indirectamente a través de los efectos multiplicadores del gasto que ellas financian;

- Por su papel en la reducción de los niveles de pobreza y desigualdad social que desde siempre han caracterizado a las sociedades en vías de desarrollo.

Lo interesante es que da la impresión que desde los organismos internacionales se estuviera impulsando un nuevo paradigma del desarrollo para ser instrumentado en nuestras sociedades, y en el cual la migración y las remesas asumirían un rol preponderante, en sustitución del rol que en anteriores esquemas y paradigmas del desarrollo habrían jugado tanto el Estado como el propio Mercado. En este nuevo paradigma, las remesas conformarían una especie de capital económico, el cual junto a otros capitales sociales (redes familiares, trabajo familiar y comunitario, organizaciones de migrantes, entre otros), constituirían recursos privilegiados para las comunidades que, si fueran bien gestionados, podrían contribuir a superar las condiciones de vulnerabilidad social y precariedad económica, aun cuando las condiciones del entorno estructural en el que viven no les sean favorables. ${ }^{1}$

En efecto, este enfoque sobre el impacto de las remesas y el papel de los migrantes en el desarrollo, es coherente con los principios que sustentan las nuevas políticas de desarrollo y combate a la pobreza impulsadas en la última década. A diferencia del carácter asistencialista que estaba impregnado en las anteriores políticas de combate a la pobreza, este nuevo enfoque traslada el eje de atención a la promoción de una correcta gestión de los activos y recursos de los pobres, para que ellos mismos enfrenten y superen su situación de pobreza y vulnerabilidad (Moser, 1998; Banco Mundial, 2004). Según este enfoque, los pobres pueden ser los protagonistas del proceso de desarrollo porque cuentan con los recursos necesarios para tal fin, entre ellos las remesas. En todo caso, tan sólo necesitan

\footnotetext{
${ }^{1}$ Los presupuestos políticos e ideológicos que sustentan estas posiciones son evidentes. De hecho, tan sólo pensar en la posibilidad de que las remesas sean la base de un nuevo paradigma del desarrollo resulta no sólo falto de sensatez académica, sino mera retórica y demagogia política. Un nuevo paradigma debe sustentarse en nuevas teorías sobre el desarrollo, así como en su confrontación con datos empíricos que las corroboren, aspectos que indudablemente, están ausentes en todos estos discursos celebratorios de las remesas y la migración que predominan en el discurso oficial de organismos internacionales de promoción del desarrollo, y de no pocos gobiernos nacionales de países con altos índices de emigración internacional.
} 
aprender a usarlos y gestionarlos correctamente. Medidas como el empowerment, el autoempleo y el aprovechamiento del capital social de los pobres constituirían mecanismos privilegiados para resolver su situación de vulnerabilidad.

De esta manera, entre las líneas estratégicas para el desarrollo, tanto de gobiernos nacionales como de organismos internacionales, figura en lugar destacado la necesidad de orientar las remesas hacia la creación de pequeñas y medianas empresas, así como hacia otro tipo de gastos que fomenten la formación de capital productivo y humano (Ratha, 2003). En el caso de México y Centroamérica, por ejemplo, esta tesis forma parte ya de los programas oficiales del gobierno, en los que el autoempleo y la promoción de negocios familiares financiados con remesas se ofrecen como alternativas al desempleo y la pobreza. Es el caso, por ejemplo, de los programas $3 \times 1$, a través de los cuales se busca fomentar la inversión productiva y la formación de negocios por parte de los migrantes y/o sus familiares. ${ }^{2}$

ii) Junto a estos textos de corte más bien político, en los últimos años han proliferado una serie de estudios econométricos que buscan dar sustento empírico a estas visiones celebratorias del papel de las remesas en el proceso de desarrollo.

Por un lado, están aquellos estudios que a través de modelos de contabilidad social, buscan estimar el impacto de las remesas a través de los efectos multiplicadores que ellas generan (Adelman y Taylor, 1990; Taylor y Wyatt, 1996; ZárateHoyos, 2007; Hinojosa, 2003). El tipo de resultados que arrojan este tipo de estudios es la estimación del multiplicador de las remesas sobre el PIB, a través del cual se puede estimar y calcular el impacto efectivo de las remesas sobre la dinámica económica.

Estos trabajos se sustentan en enfoques macroeconómicos de tipo keynesiano, que describen y explican los efectos multiplicadores que este tipo de transferencias pudieran tener sobre el ingreso y la actividad económica interna de los países perceptores. En concreto, de acuerdo con los modelos keynesianos, aún cuando las remesas (o cualquier otro tipo de transferencia externa) se gaste solamente en consumo familiar, ellas tienen un impacto multiplicador sobre el producto interno y la actividad económica. Asimismo, la teoría keynesiana señala además que la magnitud de estos efectos multiplicadores depende directamente de la propensión a ahorrar e indirectamente de la propensión a importar (compra de bienes externos). Posteriormente, Albert Hirschman extendió estos modelos en términos de las posibilidades de crecimiento y desarrollo económico que ellos pudieran generar bajo determinadas circunstancias.

\footnotetext{
${ }^{2}$ Los programas $3 \times 1$ se basan en los principios del codesarrollo, y se sustentan en la concurrencia de fondos entre diversas instancias gubernamentales y de los propios migrantes. De esta forma, en el caso mexicano por ejemplo, el programa $3 \times 1$ consiste en que por cada dólar que aportan los migrantes, el gobierno federal, el gobierno estatal y el gobierno municipal aportan cada uno otro dólar adicional. Con este fondo concurrente se financiarían tanto proyectos productivos privados como obras de infraestructura social. Sobre los logros y limitaciones de estos programas, véase Delgado Wise et al., 2004; Torres, 2001.
} 
Por otro lado, y ante el creciente interés de considerar a las remesas de los migrantes como un instrumento de desarrollo de los países emisores, especialmente del Tercer Mundo, en los últimos años han proliferado una serie de estudios basados en modelos econométricos, que buscan medir el impacto de las remesas en diversos ámbitos de la economía nacional, a saber: en la distribución del ingreso, reducción de la pobreza, impulso al crecimiento económico, formación de capital humano, entre otros.

Así por ejemplo, Adams, Richard y Page (2005), estiman que un incremento del $10 \%$ de la tasa de emigración (con sus consecuentes incrementos de las remesas) generan una reducción de $2.1 \%$ en la tasa de pobreza de cada país, medida esta última como la proporción de población que vive con menos de un dólar diario. Por su parte, un estudio del FMI (2005) encuentra que un incremento de la relación remesas/PiB de 2.5 puntos porcentuales está estadísticamente asociada con una caída del $0.5 \%$ de la tasa de pobreza. Asimismo, Acosta et al. (2006), extienden estos modelos a modo de estimar la magnitud del impacto de las remesas según el nivel de desarrollo y pobreza de cada país. En concreto, estos autores encuentran que un incremento del 10\% en la participación de las remesas sobre el PIB de cada país, implicaría la reducción del nivel de pobreza entre un $0.04 \%$ (países pobres) y un $0.5 \%$ (países ricos).

En general, en estos trabajos más que intentar demostrar tal o cual teoría que explique la relación Remesas-Desarrollo, lo que buscan es dar sustento econométrico a un discurso político preestablecido, a través de diversas mediciones empíricas sobre el impacto de las remesas en tal o cual variable macroeconómica. De hecho, y parafraseando a Sami Naïr (2006), podemos afirmar que el saber generado por este tipo de estudios es con frecuencia más valioso para comprender los intereses políticos subyacentes a este tipo de discursos, que para entender y dimensionar los impactos económicos de las remesas.

En efecto, el principal resultado al que llegan estos estudios, no es en términos de describir las causas y mecanismos de por qué las remesas tienen tal o cual impacto económico, objetivo, sin duda, académico y de conocimiento científico, sino más bien, obtener estimaciones y medidas del tipo en cuánto se reduciría la pobreza o la desigualdad, en cuánto aumentaría el PIB, ante un determinado aumento del volumen de las remesas, objetivo, sin duda, más cercano a una estrategia de política económica con el conjunto de intereses y prioridades que ella implica.

Ahora bien, tanto en el caso de estos estudios que buscan medir los efectos multiplicadores como aquellos que buscan medir otros impactos macroeconómicos de las remesas, ambos presentan ciertas limitaciones metodológicas y conceptuales que quisiéramos comentar.

Por un lado, a nivel metodológico, ambos tipos de estudios suelen centrarse en la estimación de parámetros que midan los efectos económicos de las remesas, sin que paralelamente ofrezcan información o estimaciones sobre el valor del 
mismo parámetro, pero para otras variables macroeconómicas. Al no disponerse de estas estimaciones alternativas, no siempre es posible saber si el efecto multiplicador de las remesas es realmente importante, o si por el contrario, es muy inferior al efecto que se obtendría a partir de otras categorías macroeconómicas.

En otras palabras, estos modelos no nos indican si el valor del coeficiente de las remesas es mayor o menor que el de otras variables. Así por ejemplo, en algunos casos podemos saber que un incremento de un $1 \%$ de las remesas puede contribuir a reducir la pobreza en un $0.5 \%$, por ejemplo, pero no sabemos si ese efecto es mayor o menor al que se lograría con un incremento de un 1\% del PIB, o de un $1 \%$ de las transferencias públicas a través de programas sociales de combate a la pobreza, etc.

Por otro lado, a nivel conceptual, la debilidad de estos estudios parece aún mayor. En efecto, al igual que los discursos de los organismos internacionales, estos estudios econométricos presentan un importante vacío conceptual al momento de dar sustento teórico a las relaciones que se analizan, ya sea empíricamente, en unos casos, o de las relaciones que se promueven políticamente, en otros.

En efecto, en casi todos estos estudios la pregunta que da sentido a la argumentación que se plantea no es por qué las remesas tienen un impacto económico (del tipo que fuera), sino que se restringen a analizar cómo se daría y cómo se potenciaría ese hipotético impacto.

En el caso del discurso y de los textos que difunden los organismos internacionales, por ejemplo, la argumentación del cómo es en favor de una perspectiva política del desarrollo. Los discursos oficiales no suelen argumentar los por qué, es decir, los argumentos lógicos y teóricos que sustenten una política de desarrollo a partir de las remesas, sino que argumentan a favor de una posibilidad, de una oportunidad económica que podrían representar las remesas. En general, estos textos suelen analizar el impacto de las remesas bajo el supuesto de si ellas se destinaran a un uso más productivo, ya sea para financiar inversión privada, o para la formación de capital humano, o para infraestructura social, entre otros. Sin embargo, no ofrecen una argumentación lógico-conceptual que explique y sustente por qué las remesas podrían o deberían tener tales fines.

La trampa teórico-metodológica de este tipo de argumentación es obvia. Cualquier recurso que destinemos a inversión productiva tendrá efectos sobre el desarrollo económico. Eso es obvio, y no está en discusión. La cuestión de fondo es por qué son las remesas las que tienen que destinarse a esos fines, y no otros recursos. La respuesta a esta pregunta puede hacerse desde dos ámbitos: o bien se dan argumentos políticos que justifiquen esta opción (responsabilidad de los sujetos de su desarrollo, etc.), o bien se dan argumentos teóricos que expliquen y sustenten esta vocación de las remesas. Los discursos oficiales suelen centrarse en el primer ámbito, dejando muchos vacíos y silencios respecto al segundo. 
Por su parte, los estudios empíricos basados en modelos econométricos tampoco se sustentan en modelos teóricos, sino que buscan más bien dimensionar empíricamente ese supuesto impacto de las remesas que argumentan y señalan los discursos oficiales. Estos trabajos no sólo son funcionalistas, como los califican Jones (1998) y Binford (2002), sino que además podemos decir que muchos de ellos son lisa y llanamente empiricistas. Curiosamente, estos trabajos no buscan la demostración de una teoría de las remesas, sino medir y demostrar un supuesto impacto de las remesas, el cual no ha sido conceptualizado ni teorizado. ${ }^{3}$

Considerando estas deficiencias teóricas y metodológicas de los estudios, nuestra propuesta busca enfocar el tema de la relación Remesas-Desarrollo precisamente a partir de su posible conceptualización con base en los marcos teóricos que podrían darle sustento. En este sentido, la pregunta básica que orienta nuestra reflexión no es determinar o dimensionar cuál es el impacto de las remesas, sino una más básica: ipor qué las remesas debieran tener algún impacto sobre la dinámica de desarrollo de las economías del Tercer Mundo?

Aun a riesgo de caer en un excesivo positivismo, nuestro interés es discutir los marcos teóricos que permitan sustentar este tipo de relación, y desde los cuales podamos configurar hipótesis concretas que puedan ser demostradas o refutadas empíricamente. Al respecto, un primer punto a resolver es definir conceptualmente las remesas. Generalmente los diversos trabajos parten de una definición operativa, de cómo ellas son medidas en la Balanza de Pagos de cada país, o de las recomendaciones que al respecto hacen organismos internacionales (Carling, 2007). Nuestro punto de partida es otro. Antes de su definición operativa, es necesario plantear el marco conceptual desde el cual entendemos y analizamos tanto las remesas como la migración internacional en el mundo contemporáneo.

\section{MODELO MACROECONÓMICO PARA ANALIZAR EL IMPACTO DE LAS REMESAS}

Las remesas son una transferencia de recursos entre privados, el punto en discusión es definir su carácter y significado económico (además de sus significados sociales y culturales). Como flujo de transferencias, las remesas tienen un impacto en la dinámica macroeconómica y microeconómica de los países perceptores. No obstante, este impacto dependerá directamente del significado económico que ellas asuman en cada momento.

Al respecto, desde la macroeconomía podemos recuperar algunos modelos analíticos que nos permitan entender y dimensionar el peso e impacto de las remesas, dependiendo del carácter específico que ellas asuman. Analíticamente, con base en estos modelos podemos establecer una distinción entre dos grandes cate-

3 Sólo en el caso de los estudios sobre los efectos multiplicadores, se parte de un marco conceptual reconocido. 
gorías: por un lado, las llamadas remesas salariales (familiares), y por otro las llamadas remesas de capital (productivas).

- Las primeras, corresponden a transferencias directas para su uso familiar, ya sea para el consumo (remesas salario) para ser ahorradas en función de un consumo familiar futuro, o para casos de emergencias familiares, o bien para sustentar los gastos de la reproducción de los usos y costumbres familiares que implican la reproducción de sus relaciones culturales.

- Las remesas productivas en cambio, corresponden a diversas formas de inversión privada o social, que no pasan por el presupuesto familiar (señalar estas posibles inversiones).

Esta distinción básica no es casual, se deriva de un marco conceptual macroeconómico el cual nos permite conceptualizar y dimensionar los posibles impactos de una y otra categoría de remesa. En efecto, desde la teoría macroeconómica podemos identificar el ámbito de incidencia y los posibles impactos de cada tipo de remesa, en función, precisamente, de su particular función como categoría económica.

En el caso de las remesas salariales, por ejemplo, ellas contribuyen a sustentar el balance ingreso-gasto de los hogares. Por ese medio, sus impactos se dan en dos sentidos diferentes y complementarios.

- Por un lado, por su aporte al consumo familiar, contribuyen a elevar el nivel de vida y bienestar de los hogares perceptores, a la vez que inciden en la dinámica de las desigualdades económicas y las condiciones de pobreza.

- Por otro lado, esta misma contribución al gasto de los hogares se traduce en efectos multiplicadores hacia el resto de la economía local, regional y nacional. No obstante, el mismo modelo nos indica también en forma muy clara y precisa, que no debemos confundir estos efectos multiplicadores y de bienestar de las familias, con los impactos que las remesas pudieran tener directamente como instrumento de desarrollo.

Por su parte, en el caso de las remesas de capital, desde la macroeconomía se señala que su aporte viene dado a través de su contribución al balance ahorro-inversión. Como fuente de inversión, sí podemos considerar a las remesas productivas como un instrumento de crecimiento económico, que junto a otros fondos de inversión (inversión extranjera directa, inversión doméstica privada, inversión pública, etc.) son la base de todo proceso de desarrollo.

Se trata no sólo de categorías distintas en cuanto a su origen, sino especialmente en relación a su función y significado económico para los países que las perciben. En efecto, no podemos confundir este impacto de las remesas de capital en el crecimiento económico con un hipotético impacto en el bienestar de la población y/o reducción de la pobreza, el cual está más bien asociado a las remesassalariales. 
Considerando estas distinciones en torno al significado económico de las remesas, a continuación presentamos un modelo de análisis que a la vez que contextualiza, permite ilustrar las relaciones macroeconómicas asociadas a cada categoría de remesas (salarial o de capital) y a partir de las cuales podemos conceptualizar y estimar los posibles impactos económicos de cada una de ellas.

Modelo de análisis del impacto económico de las remesas

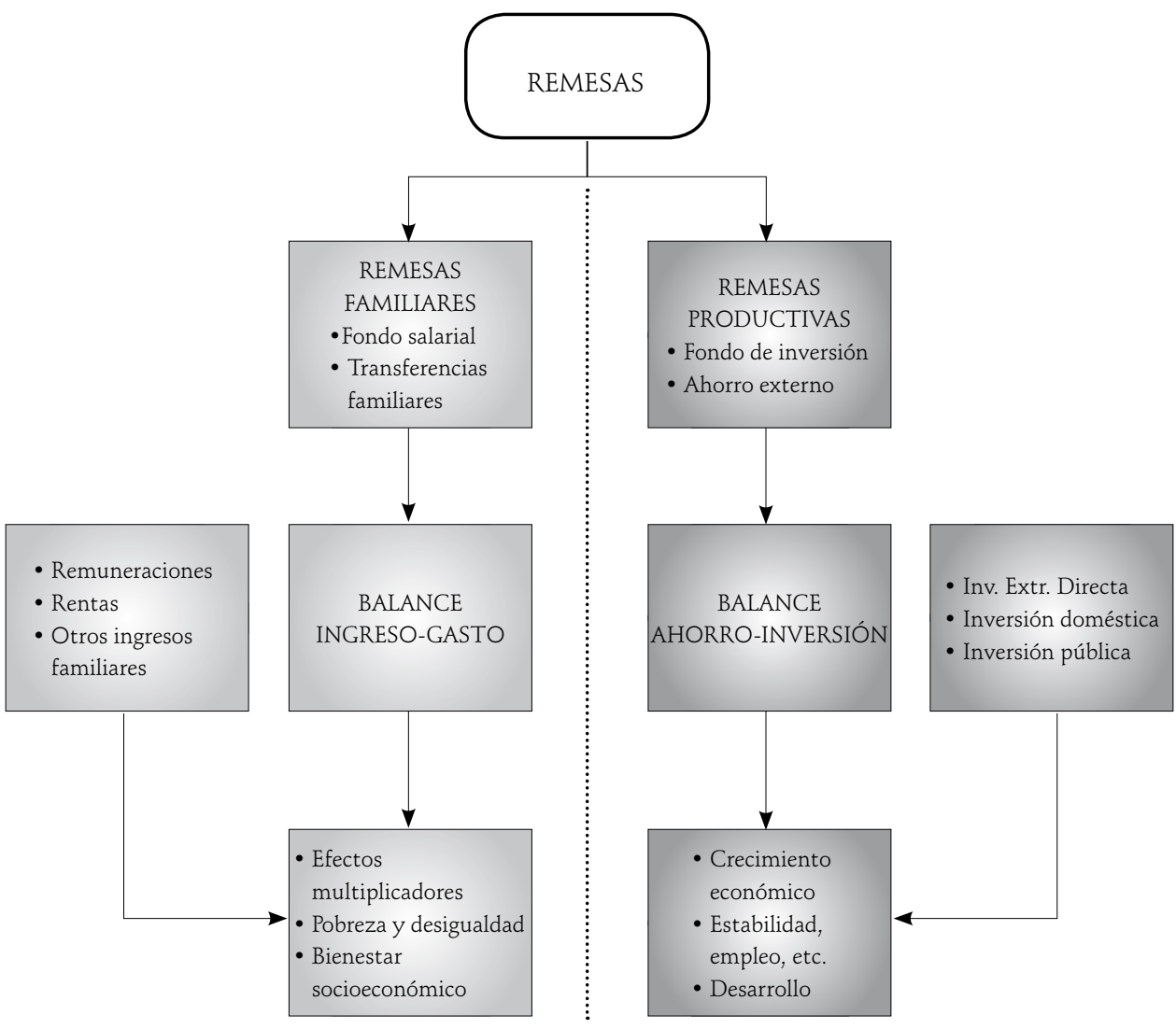

Lo relevante de este modelo analítico-conceptual, es que por un lado, se basa en la teoría macroeconómica, y por otro, nos ofrece un marco de entendimiento de los impactos de las remesas, de acuerdo a las diferentes modalidades o categorías concretas de remesas.

- Por un lado, nos permite entender cómo y por qué las remesas salariales pueden contribuir (o no) a reducir la pobreza de los hogares perceptores, o bien a incidir en la distribución del ingreso y niveles de bienestar de la población.

- Por otro lado, nos permite entender cómo y por qué las remesas de capital pueden contribuir (o no) a sustentar un proceso de crecimiento económico y estabilidad macroeconómica.

- Pero también, este modelo nos señala que no podemos confundir un efecto con otro, o lo que es lo mismo, que el impacto concreto de cada remesa está en función 
de su papel como categoría económica, esto es, de su particular definición conceptual, la cual se deriva directamente de la modalidad concreta de remesa que se trate en cada momento y lugar.

- Asimismo, y como corolario de lo anterior, este modelo analítico no está necesariamente reñido con algunas de las propuestas más generalizadas sobre la relación Remesas-Desarrollo, sino que contribuye a generar un marco de entendimiento de ellas. Así por ejemplo, este modelo reconoce los ámbitos de acción de los efectos multiplicadores, así como sus alcances y limitaciones.

- Por último, esta distinción, si bien nos permite configurar un marco de entendimiento de las remesas que incluye diversas modalidades con distintos efectos, no implica necesariamente que cada uno de estos efectos sea por definición económicamente importante y de gran magnitud. El modelo sólo nos indica los ámbitos de acción e incidencia de cada modalidad de remesa. No obstante, con base en este modelo, podemos diseñar estrategias metodológicas que nos permitan medir y dimensionar el impacto específico de cada modalidad de remesas respecto a cada ámbito macro y microeconómico en cuestión.

Ahora bien, el anterior es esencialmente, un modelo analítico, no teórico. Es decir, nos permite identificar los mecanismos y relaciones cómo las diferentes categorías de remesas, en tanto transferencias externas privadas, impactan en la dinámica económica. Pero aún no señalan el por qué de esta relación e impacto. Asimismo, este modelo no señala por qué las remesas configuran uno u otro tipo de modalidad de transferencia de recursos (transferencia salarial v/s transferencia de capital). Sólo señala los mecanismos en que una y otra categoría de remesas puede impactar sobre la dinámica económica.

Para comprender y explicar estos impactos, es necesario entonces partir de una teoría de las remesas, esto es, de un marco conceptual que las explique, defina y aprehenda, y desde el cual podamos describirlas y definirlas como uno y/u otro tipo de transferencia de recursos. En otras palabras, necesitamos un marco conceptual que explique por qué las remesas son un tipo de transferencia salarial, o por el contrario, por qué serían un tipo de transferencia de capital, o bien por qué son de ambos tipos. En síntesis, necesitamos un marco conceptual que nos permita responder a la más sencilla de las preguntas: iqué son las remesas?

Sabemos que las remesas son transferencias internacionales, que fluyen desde los migrantes hacia sus familias y comunidades de origen. Conocemos y entendemos las vicisitudes de su definición operativa, y la dificultad metodológica de medir y registrar este tipo de transferencias privadas. Sin embargo, sabemos también que el problema no está allí, sino en la conceptualización de las remesas como categoría económica y social. Al respecto, podemos plantear diversas interrogantes, a saber:

- iSon las remesas un fondo de ahorro-inversión privada o configuran un fondo salarial para financiar el consumo privado? 
- En uno y otro caso (como fondo de inversión o como fondo salarial), żcuáles son los impactos que la teoría macroeconómica plantea para ese tipo de transferencias externas?

- ¿Qué tipos o modalidades de remesas existen en función de sus impactos sobre la dinámica económica?

- ¿Cuál es el peso real de cada una de ellas?

Para responder estas y otras preguntas sobre la función e impacto económico de las remesas, creemos necesario partir de una teoría de alcance mayor que permita entender y explicar la dinámica y significado de las migraciones internacionales en la sociedad actual. Esta teoría debiera servirnos como marco contextual y de referencia para conceptualizar y definir no sólo la función económica de las remesas, sino también para establecer los ámbitos y dimensiones de sus posibles impactos en otras variables macroeconómicas (crecimiento, pobreza y bienestar, distribución del ingreso, entre otras).

\section{MIGRACIÓN Y REMESAS EN LA SOCIEDAD POSTINDUSTRIAL}

Es común que en los discursos de gobiernos nacionales y organismos internacionales, el ámbito de análisis de las remesas se circunscriba a sus hipotéticos impactos en las sociedades y economías que las perciben, sin considerar el contexto global que genera y explica la dinámica migratoria en el mundo actual. En particular, las remesas son vistas como un recurso dado del que no se cuestiona su origen (salarial, trabajo precario y flexible, globalización económica, entre otros). Ello no es casual, sino que refleja la debilidad conceptual y los prejuicios ideológicos que sustentan a esos discursos.

En efecto, el silencio que envuelve a las condiciones de origen de las remesas, especialmente en términos de las condiciones de exclusión social y segregación laboral que caracteriza la inserción laboral de los migrantes, no es un fenómeno casual, sino que es resultado de una peculiar visión de la migración y las remesas. Si el análisis se centra en el papel de las remesas en las sociedades que las perciben, es porque se asume implícitamente a veces, explícitamente en otras, que el problema está en los países de origen de la migración, especialmente en las condiciones estructurales que caracterizan su subdesarrollo y que dan origen a la emigración de fuerza de trabajo.

De esta forma, la migración y las remesas se ven como una consecuencia del subdesarrollo, no como una manifestación de la globalización económica que integra y subordina estas regiones de emigración a la economía global y postindustrial. Asimismo, la migración y las remesas se ven también como una oportunidad para estas economías subdesarrolladas, como un recurso que si fuera bien gestionado, permitiría superar las condiciones estructurales de precariedad, pobreza y 
desigualdad que generaron la emigración laboral. Así, ante la falta de crecimiento económico y de fuentes de inversión, las remesas se convierten en una inusual alternativa, ya sea a través de sus efectos multiplicadores, o directamente como fuente de financiamiento de la inversión productiva. Ante las condiciones de pobreza y precariedad social, las remesas se levantan como una oportunidad de ingresos que permitiría mejorar los niveles de bienestar de la población, y de ese modo superar su condición de pobreza. ${ }^{4}$

Nuestra visión, en cambio, parte de una perspectiva más comprensiva, en donde no sólo importan las condiciones de los países expulsores de mano de obra, sino también y especialmente la de los países perceptores de esta migración laboral. Es una visión que intenta integrar los procesos migratorios actuales en la dinámica de las transformaciones estructurales que genera la globalización de la economía mundial. En esta perspectiva, la migración ya no es sólo un problema del y para el Tercer Mundo, sino que es un fenómeno consustancial a la globalización, y por lo mismo, debe incorporar también los fenómenos y transformaciones de las economías de los países desarrollados y altamente industrializados, transformaciones que inciden directamente en la configuración de los procesos migratorios y de los flujos de remesas en el mundo contemporáneo.

En este sentido, el significado social y económico de las remesas en el mundo actual no puede entenderse sin considerar simultáneamente el carácter y significado que asume la migración internacional en la actualidad. Asimismo, de acuerdo a diversos autores, la migración internacional en el mundo contemporáneo no puede entenderse si no se consideran los cambios estructurales que la globalización de la economía mundial ha generado en el sistema de relaciones económicas internacionales (Castles y Miller, 1993; Naïr, 2006; Sassen, 1998).

En efecto, con el proceso de globalización se activan diversos mecanismos de inclusión y exclusión social que, a través de la precarización del empleo y otras formas modernas de segregación social, han dado origen a un nuevo patrón de polarización y diferenciación social basado en dos procesos diferentes y complementarios.

- Por un lado, la configuración de un régimen laboral basado en los procesos de flexibilidad y desregulación, y que da origen a lo que Beck (2000) denomina como régimen de riesgo laboral, el cual sustituye el anterior régimen laboral e instituciones sociales característicos del Estado de Bienestar.

\footnotetext{
${ }_{4}$ Resulta paradójico pero igualmente revelador, que en estos discursos no se cuestionen las causas de la desigualdad, ni de la pobreza ni del subdesarrollo y bajo crecimiento económico, sino que pura y simplemente se toman como factores estructurales dados. Por el contrario, el énfasis es puesto en la oportunidad que representa este gran flujo de remesas para enfrentar y resolver al menos en parte estos problemas estructurales. Se establece así un sospechoso silencio tanto sobre las causas estructurales del subdesarrollo, de la pobreza y de la desigualdad, como sobre las responsabilidades de gobiernos y organismos internacionales en la aplicación de las políticas de ajuste estructural que contribuyeron no sólo a estructurar estas condiciones de bajo crecimiento, pobreza y desigualdad social, sino que además, son la base estructural que explica en gran medida el auge de la emigración desde estos mismos países.
} 
- Por otro lado, la transformación del sistema de ocupaciones, con base en la creciente segmentación y polarización de ellas y la diferenciación social que implican (Castells, 1998, Pioré, 1979).

Esta degradación de las condiciones laborales termina expulsando a la mano de obra local de aquellos puestos de trabajo que han sido flexibilizados y desregulados, siendo reemplazada por fuerza de trabajo migrante, que con base en su condición de vulnerabilidad es posible ser contratada en peores condiciones laborales, muchas veces sin posibilidades de sindicalizarse, sin contratos, con bajos salarios y con una alta inestabilidad laboral (Zlolniski, 2006; Sassen, 1998). Nos referimos a empleos precarizados y desvalorizados, como limpieza y mantenimiento de edificios, jardineros, lavaplatos, empleados en restaurantes, servicio doméstico y de cuidado de personas, y otras ocupaciones similares en los que se da una creciente incorporación de mano de obra migrante.

Con base en estos factores de diferenciación social e inserción desigual en el mercado de trabajo, se configuran grupos de población con niveles distintos de vulnerabilidad social, situación que se agrava por un contexto estructural en el que los mecanismos de negociación política y social que surgieron en la sociedad industrial, y que tomaron forma en el Estado de Bienestar, han dejado de operar para los grupos más vulnerables (Beck, 2000). Este es el mecanismo por el que en la sociedad global, se crean y recrean minorías sociales y culturales, como los inmigrantes, cuya vulnerabilidad construida socialmente se traslada al mercado laboral, bajo la forma de una desvalorización de su fuerza de trabajo y de sus condiciones de vida y reproducción social. ${ }^{5}$

En este contexto podemos entender el significado económico y social de las remesas. Si las migraciones constituyen un sistema de transferencia de fuerza de trabajo en un mundo globalizado desde el Tercer Mundo hacia las economías del mundo desarrollado, las remesas representan un sistema de transferencias salariales no sólo en sentido inverso, sino consustancial a este proceso global que enmarca las migraciones internacionales en la actualidad, ello al menos en un doble sentido.

- Por un lado, las remesas son una fracción de los salarios y remuneraciones de la Fuerza de Trabajo migrante en esos mercados globales. Son una parte del pago que perciben los migrantes laborales, la cual por lo mismo, tiene la misma función macroeconómica que cualquier otro salario: la reproducción de la Fuerza de Tra-

\footnotetext{
${ }^{5}$ En este marco, la tesis que sostenemos es que la pobreza y precariedad de los trabajadores migrantes no son el resultado de su exclusión del mercado de trabajo, sino que al contrario, son la forma en que ellos se integran en el mundo laboral. En el actual marco de desregulación económica y flexibilidad laboral, la modernización genera y reproduce sus propias formas de pobreza y precariedad, y en donde la condición de vulnerabilidad social de los individuos, su condición de minoría social, demográfica y cultural construida con base en sus condiciones de género, etnia, migración, deja de ser el factor de riesgo que los expone a una posible exclusión económica, para convertirse en la condición necesaria para su inclusión.
} 
bajo. Lo peculiar en este caso, es que la reproducción de la Fuerza de Trabajo se da en contextos binacionales y en mercados laborales globalizados, que se sustentan en la configuración de comunidades y familias transnacionales.

Las remesas son entonces la forma en que esta fracción del salario del migrante se transfiere a sus familias y comunidades de origen para la reproducción social de la familia y la comunidad, como lo hace cualquier otro ingreso salarial en esas mismas comunidades (u otras comunidades). Las remesas son, que duda cabe, un fondo salarial, ese es su significado y función como variable macroeconómica.

- Por otro lado, las remesas no sólo forman parte del proceso de reproducción transnacional de la fuerza de trabajo migrante, sino también de las condiciones estructurales de exclusión social y precarización laboral que enfrenta esta fuerza de trabajo. En otras palabras, al analizar el significado económico y social de las remesas no podemos hacer abstracción de este contexto global de exclusión y segregación social que caracteriza la inserción laboral de los migrantes, pues ellas (las remesas) son un producto directo de ese contexto global.

Este marco estructural de exclusión y segregación social de los migrantes, es en definitiva, lo que permite diferenciar entre el carácter y significado social de las remesas que perciben los países en vías de desarrollo, de aquellas que perciben las economías industrializadas del Primer Mundo. ${ }^{6}$

En otras palabras, las remesas no sólo constituyen un fondo salarial, sino que corresponden a ingresos salariales de trabajadores que combinan una inserción laboral de alta vulnerabilidad y precariedad en Estados Unidos, con una condición de pobreza, marginación y vulnerabilidad social en sus países de origen.

En tal sentido, las remesas fluyen de trabajadores precarios y vulnerables, hacia sus familiares que viven en condiciones de pobreza y contextos de marginación social. En este contexto, no es raro que, por un lado, las remesas se orienten fundamentalmente a financiar el consumo familiar, contribuyendo a mantener un mínimo nivel de vida, a la vez que por otro lado, no fluyan en los montos y volúmenes necesarios para promover un verdadero proceso de movilidad social ${ }^{7}$ (el siguiente esquema permite ilustrar esta idea).

Ahora bien, con base en este modelo conceptual, podemos distinguir al menos dos tipos de remesas, ambas con un mismo origen: las remuneraciones laborales de los migrantes, y una misma función económica; la reproducción social de los migrantes, sus familias y sus comunidades de origen.

${ }^{6}$ Cabe señalar que hasta el año 2000, aproximadamente, más del 40\% del flujo mundial de remesas tenía como destino los países altamente desarrollados, y era generada por sus migrantes de alta calificación que se insertan en diversos enclaves económicos que estos países mantienen en las economías del Tercer Mundo (CEPAL, 2006).

7 En un estudio reciente la CEPAL documenta el bajo monto absoluto de las remesas que perciben los hogares en América Latina. Así, por ejemplo, y considerando únicamente a los hogares perceptores de remesas, se estima que en Centro América, la remesa per cápita mensual que percibían estos hogares en el 2002 fluctuaba entre los 18 dólares (Nicaragua) y los 45 dólares (El Salvador). Asimismo, el mismo estudio documenta además, que en todos los países de la región este volumen de la remesa per cápita es muy inferior a la línea de pobreza definida en cada país por los organismos oficiales correspondientes. Para más detalles, véase CEPAL, 2005. 
Por un lado, identificamos aquellas remesas que se orientan a financiar diversos aspectos de la reproducción familiar. Entre estas remesas, podemos identificar las que financian el consumo cotidiano (consumo presente), las que permiten la compra de bienes de consumo durable, las que contribuyen a financiar gastos imprevistos o emergencias familiares de salud, deudas, entre otras, y aquellas que constituyen una forma de ahorro presente para financiar el consumo en momentos futuros, así como aquellas que podrían representar la conformación de un capital familiar, como lo es el financiamiento de la educación de los hijos, o la construcción, remodelación y/o compra de una vivienda.

Por otro lado, identificamos aquellas remesas que se orientan más bien a financiar diversos aspectos y dimensiones de la reproducción social de las familias y sus comunidades. Entre estas remesas, podemos nombrar por un lado, aquellas que se destinan a financiar el gasto de formas y relaciones ceremoniales familiares, como el gasto de fiestas familiares (quince años, bodas, bautizos, entre otras), y por otro lado, aquellas que se destinan al financiamiento de formas y relaciones ceremoniales de la comunidad, como lo son las fiestas religiosas, fiestas comunitarias, gastos de infraestructura social, entre muchas otras.

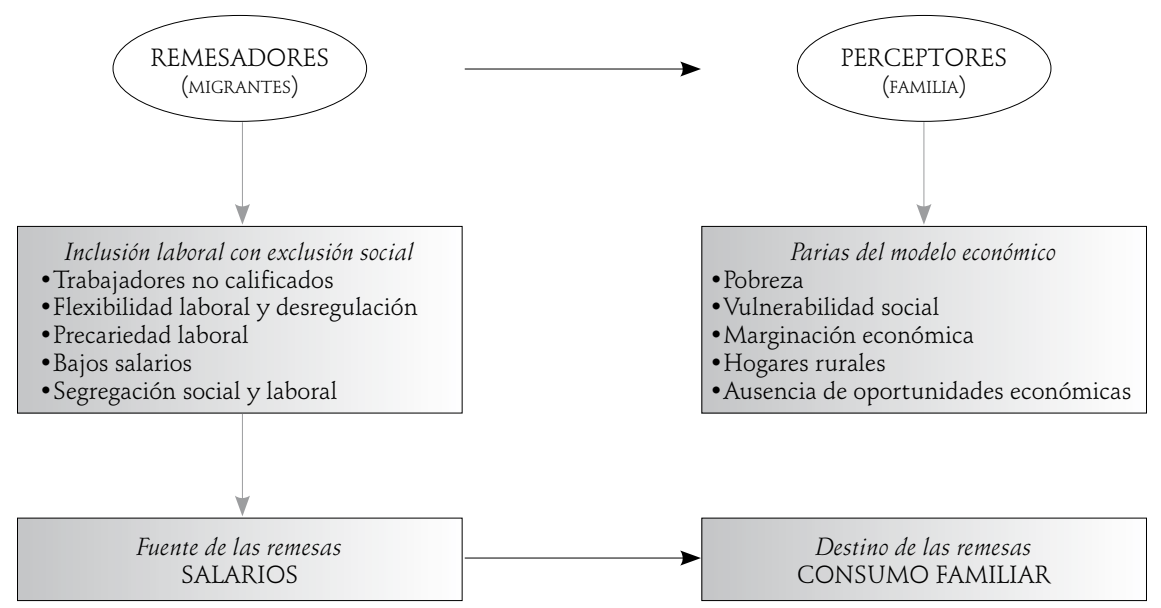

Ahora bien, este esquema conceptual permite dar sustento teórico a un enfoque de las remesas según el cual, ellas corresponden básicamente a la forma en que el salario generado por los migrantes es transferido y canalizado hacia sus familias en sus comunidades de origen.

Sin embargo, entendemos que no es el único enfoque sobre las remesas. Hay quienes sostienen que las mismas conforman también un fondo de inversión (transferencia de capital, o remesas-capital). La principal limitación de este enfoque, es que no parte de un marco conceptual más amplio, desde el cual se dé sustento teórico a esta definición de las remesas como transferencia de capital.

Por su significado y función económica, podemos definir a este tipo de remesas como una transferencia de capital la que por definición, tiene un impacto directo 
sobre la actividad productiva, a diferencia de las remesas-salario, las que como hemos visto, tienen un efecto indirecto. No obstante, no disponemos de enfoques globales desde los cuales poder analizar y explicar este tipo de remesas-capital. A diferencia de las remesas-salario, cuyo significado conceptual era derivado de una teoría general de las migraciones internacionales en la sociedad contemporánea, este tipo de remesas-capital no parecen tener un marco teórico de referencia.

En efecto, el origen mismo de las remesas-capital no es el mismo de la remesasalario. En este último, disponemos de un marco estructural que da sentido y significado a la remesa y la migración en la sociedad contemporánea. En el caso de las remesas-capital, pareciera tratarse de un concepto ambiguo y difuso que involucra muchas categorías y situaciones disímiles.

En este caso su origen ya no parece ser relevante, a diferencia de lo que veíamos en el caso del enfoque de las remesas como un fondo salarial. De hecho, el carácter de capital, esto es, de fondo de inversión productiva, se define en función del país que las percibe, no del país que las remite. En el caso de las remesas-salario, en cambio, el marco teórico desde el cual se partía, suponía que su concepto y significado era el mismo en ambos casos, esto es, que en su origen la remesa formaba parte del salario o remuneraciones al trabajo ganado por los migrantes, a la vez que en su uso, las remesas cumplen la misma función que cualquier otro ingreso salarial, en términos de sustentar la reproducción social de la familia.

En el caso de las remesas-capital, parece tratarse más bien de procesos individuales de difícil generalización, y por lo mismo, en torno a los cuales es mucho más compleja la formulación de alguna ley de validez general que explique este tipo de transferencias. El proceso migratorio que las genera parte de una mayor variedad de factores explicativos y desencadenantes. No sólo se trata de factores estructurales, sino también de elementos individuales y casuísticos que resultan mucho más complejos de modelar conceptual y teóricamente.

A diferencia de la teoría de las migraciones laborales, en este caso no parecen tener la misma relevancia las condiciones laborales, contractuales y estructurales de la acumulación en los países de destino de la migración. Asimismo, adquieren mayor relevancia factores individuales relacionados con la actitud empresarial del migrante, su perfil socioeconómico, etc., lo que los diferencia del modelo clásico de migrante y de migración laboral.

En este caso, aunque los migrantes que las remiten se inserten en el mismo circuito de migración laboral global que los otros migrantes laborales, no forman parte de este flujo de migración laboral, en cuanto a sus determinantes estructurales. Su migración surge de otras causas: ausencia de recursos, créditos, y otros apoyos necesarios para financiar una empresa productiva o proyectos de infraestructura. En este caso, podemos afirmar que la migración y las remesas surgen como una oportunidad, ante la ausencia de opciones que tradicionalmente ofrecen el mercado, el Estado, onG, así como organismos internacionales y gobiernos de países desarrollados, a través de sus políticas y programas de cooperación para el desarrollo (Canales y Montiel, 2004). 
Lo relevante en todo caso, es que no hay una teorización que explique este tipo de desplazamientos y migraciones no laborales, más allá de marcos meramente descriptivos que permiten caracterizar algunos aspectos de estas migraciones, pero en ningún caso logran explicarlas ni comprenderlas, al menos no en la profundidad y sistematicidad como lo podemos hacer actualmente respecto a las migraciones laborales.

En este sentido, lo que más se ha avanzado es en una descripción de tipos o modalidades de transferencias de capital, definidas con base en su uso en las comunidades de destino, y por lo mismo, completamente desprovistas de un marco conceptual que las explique.

Al respecto, y a modo de síntesis, en la siguiente tabla presentamos, a modo de ejemplo, una tipología de las remesas que incluye tanto aquellas que hemos definido como Remesas-Salario, como las que hemos definido como Remesas de Capital.

Tipología para el análisis de las remesas

\begin{tabular}{|c|c|c|c|}
\hline \multirow{8}{*}{$\begin{array}{l}\text { Remesas-Salario } \\
\text { (Familiares) }\end{array}$} & \multirow{6}{*}{$\begin{array}{l}\text { Reproducción } \\
\text { familiar }\end{array}$} & \multirow[t]{2}{*}{ Remesa-Salario } & Bienes y servicios de consumo directo \\
\hline & & & Bienes de consumo durable \\
\hline & & $\begin{array}{l}\text { Remesas-Ahorro } \\
\text { familiar }\end{array}$ & Bienes y servicios de consumo futuro \\
\hline & & Remesas-Emergencia & Salud, imprevistos, etc. \\
\hline & & \multirow{2}{*}{$\begin{array}{l}\text { Remesas-Capital } \\
\text { familiar }\end{array}$} & Vivienda, autos, etc. \\
\hline & & & Educación \\
\hline & \multirow[t]{2}{*}{$\begin{array}{l}\text { Reproducción } \\
\text { social }\end{array}$} & $\begin{array}{l}\text { Remesas-Gasto } \\
\text { familiar }\end{array}$ & $\begin{array}{l}\text { Fiestas y ceremonias familiares } \\
\text { (quince años, bodas, bautizos, } \\
\text { funerales, etc.) }\end{array}$ \\
\hline & & $\begin{array}{l}\text { Remesas-Gasto } \\
\text { comunitario }\end{array}$ & $\begin{array}{l}\text { Fiestas y ceremonias comunitarias } \\
\text { (religiosas, sociales, etc.) }\end{array}$ \\
\hline \multirow[t]{5}{*}{$\begin{array}{l}\text { Remesas-Capital } \\
\text { (productivas) }\end{array}$} & \multirow[t]{2}{*}{$\begin{array}{l}\text { Remesas-Inversión } \\
\text { privada }\end{array}$} & $\begin{array}{l}\text { Inversión negocios } \\
\text { urbanos }\end{array}$ & $\begin{array}{l}\text { Capital fijo, capital de trabajo, } \\
\text { reinversiones, pago de impuestos, etc. }\end{array}$ \\
\hline & & $\begin{array}{l}\text { Inversión predios } \\
\text { agrícolas }\end{array}$ & $\begin{array}{l}\text { Tierra y maquinaria, insumos, } \\
\text { ganado, etc. }\end{array}$ \\
\hline & $\begin{array}{l}\text { Remesas-Ahorro } \\
\text { privado }\end{array}$ & $\begin{array}{l}\text { Remesas-Ahorro } \\
\text { especulativo }\end{array}$ & $\begin{array}{l}\text { Depósitos en bancos, Tandas, } \\
\text { Préstamos, etc. }\end{array}$ \\
\hline & $\begin{array}{l}\text { Remesas-Inversión } \\
\text { colectiva }\end{array}$ & $\begin{array}{l}\text { Ahorro-Inversión } \\
\text { productiva social }\end{array}$ & $\begin{array}{l}\text { Infraestructura productiva, } \\
\text { comunicaciones y transportes, etc. }\end{array}$ \\
\hline & $\begin{array}{l}\text { Remesas-Inversión } \\
\text { social }\end{array}$ & $\begin{array}{l}\text { Remesas-Inversión } \\
\text { social }\end{array}$ & $\begin{array}{l}\text { Bienes públicos, infraestructura social, } \\
\text { parques y jardines, etc. }\end{array}$ \\
\hline
\end{tabular}

\section{DETERMINANTES MACROECONÓMICOS DE LAS REMESAS}

Aun cuando estas transferencias de capital no dispongan de un marco conceptual que las defina y explique, no por ello debemos descartarlas pura y simplemente. 
En términos económicos y empíricos, pueden resultar importantes, aunque no podamos explicar por qué lo son. En este sentido, lo que hemos apuntado en la sección anterior es la debilidad conceptual y teórica de los discursos dominantes sobre las remesas y sus supuestos impactos económicos. Sin embargo, queda aún por demostrar su debilidad empírica, que en realidad, constituiría una debilidad todavía mayor, en función de los intereses y propuestas que se mueven a partir de esos discursos dominantes.

Para ello, haremos uso de modelos econométricos que nos permitan estimar los factores determinantes de las remesas, y con base en ellos, demostrar cuál es el significado y carácter económico de las mismas. Si llegamos a conocer cuáles son los factores que determinan su comportamiento macroeconómico, entonces podemos saber qué tipo de categoría macroeconómica son en realidad.

Nuestra hipótesis es que las remesas constituyen esencialmente un tipo de transferencia salarial, y muy esporádica y eventualmente un fondo de ahorroinversión o transferencia de capital. De esta forma, si se trata efectivamente de una transferencia salarial, entonces es de esperar que, en tanto variable macroeconómica, su comportamiento es mucho más sensible a las condiciones socioeconómicas y de vida de la población, que a las condiciones de inversión y crecimiento económico de cada país. En otras palabras, en términos de su comportamiento macroeconómico, las remesas se asemejan mucho más a un ingreso familiar que a un fondo de ahorro e inversión productiva.

Para demostrar esta tesis, a continuación presentamos un modelo econométrico que relaciona el volumen de las remesas con diferentes variables macroeconómicas. Se trata de un modelo de regresión lineal de series de tiempo, a través del cual podemos estimar sus determinantes macroeconómicos. Hemos aplicado este modelo para cuatro países de la región (México, Colombia, El Salvador y República Dominicana), intentando abarcar diversas situaciones económicas, y que puedan representar las distintas subregiones geográficas de América Latina. Asimismo, se trata de países con altos volúmenes de remesas, y que se incorporaron al circuito remesador internacional hace más de dos décadas. Esto último es muy importante, pues nos permite tener un horizonte temporal de observación relativamente amplio, de tal manera que nos permita captar la evolución y tendencia de las remesas en diferentes contextos y situaciones macroeconómicas en cada país.

En la siguiente tabla presentamos las dimensiones y variables macroeconómicas incluidas en el modelo. La variable dependiente corresponde al volumen anual de remesas estimado por el banco central de cada país, y los datos se refieren a cada año calendario entre 1980 y 2004.

Hemos incluido variables independientes de tres tipos. Por un lado, aquellas que miden la evolución de las condiciones de vida de la población. Por otro lado, variables macroeconómicas que miden la dinámica de la economía nacional, sus 
ciclos y tendencias. Y por último, variables que miden las condiciones financieras de ahorro e inversión en cada momento.

\section{Modelo macroeconomico de las remesas}

(Modelo de serie de tiempo)

\begin{tabular}{l}
\hline \multicolumn{1}{c}{ CONDICIONES SOCIOECONÓMICAS } \\
\hline Salario mínimo en el país de origen \\
Salario mínimo en Estados Unidos \\
Inflación en el país de origen \\
\hline CONDICIONES MACROECONÓMICAS \\
\hline Tipo de cambio en el país de origen \\
Crecimiento del PIB del país de origen \\
Crecimiento del PIB de Estados Unidos \\
Índice de Competítívidad país de origen \\
\hline CONDICIONES FINANCIERAS Y EXTERIORES \\
Tasa de interés en el país de origen \\
Tasa de interés en Estados Unidos \\
Saldo de la Balanza Comercial \\
Inversión Extranjera Directa \\
Valor de las Exportaciones (fob) \\
\hline
\end{tabular}

De acuerdo a nuestra perspectiva, si las remesas son un tipo de ingreso familiar, entonces es de esperar que sean más sensibles a las condiciones de vida de la población, respondiendo también a los ciclos de cada economía. Por el contrario, si las remesas constituyen más bien un fondo de ahorro-inversión, entonces es de esperar que ellas sean más sensibles a las condiciones financieras de cada momento.

En la siguiente tabla presentamos los resultados del modelo de mejor ajuste para cada uno de los cuatro países seleccionados. Lo interesante de este modelo es que selecciona automáticamente aquellas variables que tienen un peso estadísticamente significativo en la explicación de la variable dependiente (volumen anual de las remesas en cada país), y excluye del modelo aquellas otras variables que no contribuyen significativamente a la explicación del comportamiento (varianza) de la variable dependiente.

También se presentan los resultados que arroja el modelo de mejor ajuste para cada país.

De acuerdo con estos resultados podemos establecer los siguientes hallazgos:

- En todos los casos, las condiciones socioeconómicas de la población son un factor determinante del volumen de remesas. En particular, su volumen tiende a incrementarse ante caídas del salario mínimo en los países de origen, y/o ante incrementos en el salario mínimo percibido en los lugares de destino de los migrantes. 


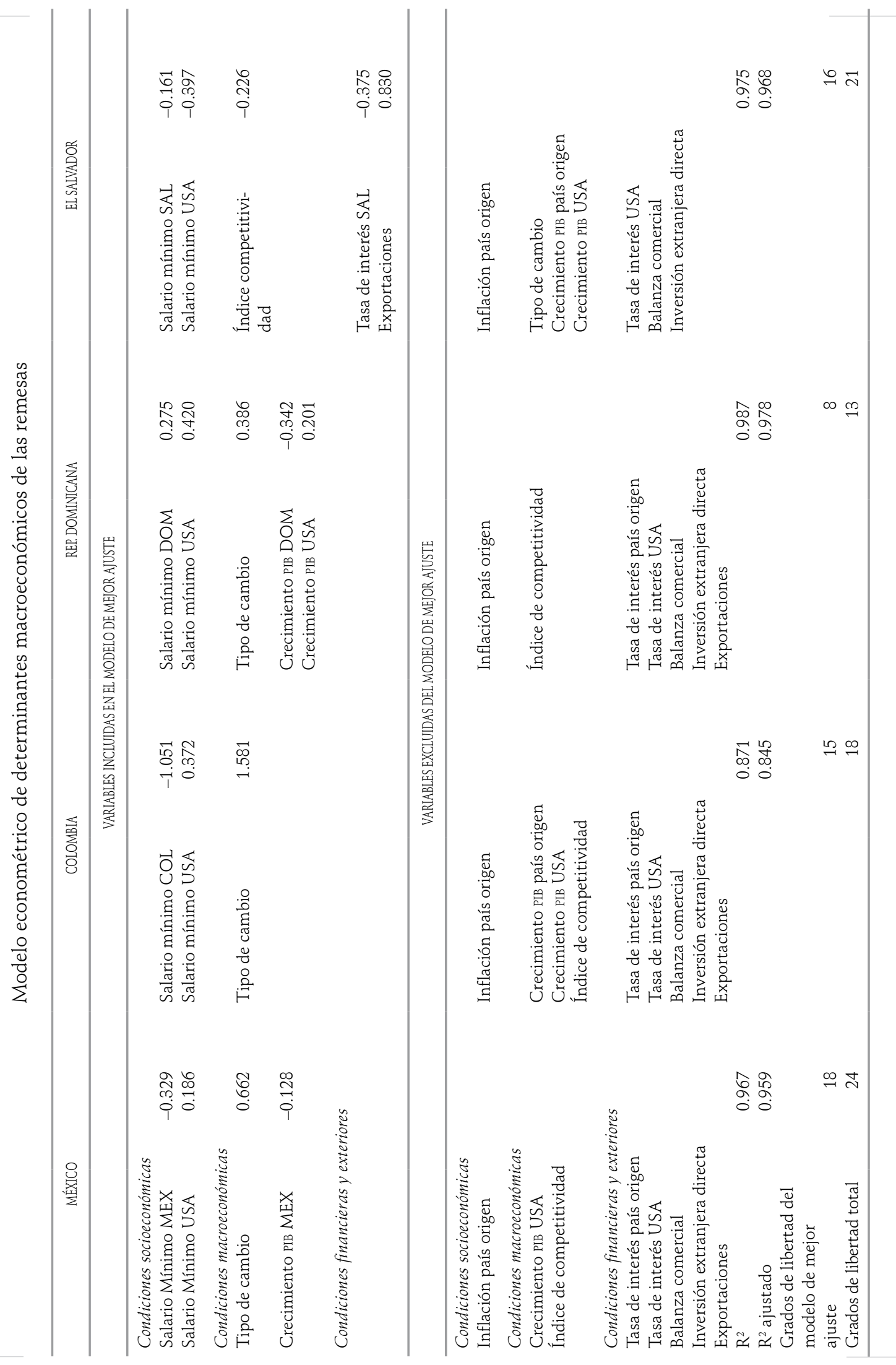


En el primer caso, ello nos indica que las remesas configuran un fondo anticíclico que permite contrarrestar los efectos negativos de las crisis en el nivel de ingresos familiares, o lo que es lo mismo, que ante caídas en el ingreso familiar (salarios), la migración y las remesas se ven como una opción viable para mantener, en la medida de lo posible, el nivel de vida y consumo familiar. En este sentido, puede inferirse que las remesas parecen contribuir a reducir los efectos de las crisis en el nivel de pobreza de la población.

En el segundo caso, el incremento del volumen de remesas ante incrementos en el salario en el lugar de destino es lo esperable, pues nos indica que éstas dependen directamente del nivel de ingresos disponible de los inmigrantes. Ahora bien, lo relevante de estas relaciones es que ellas se reproducen sistemáticamente en los cuatro países considerados en el modelo, aun cuando se trata de economías muy disímiles, cada una, además, con dinámicas muy diferentes en estos últimos 25 años.

- Por otro lado, en cuanto al papel de las condiciones macroeconómicas estructurales de cada economía, nuevamente se observa una relación sistemática que nos indica que las remesas suelen responder inversamente a la dinámica de los ciclos económicos. En efecto, el volumen de las remesas se incrementa en situaciones de crisis, caracterizadas por caídas del PIB, devaluaciones del tipo de cambio, y/o pérdidas en los niveles de competitividad externa.

Estos resultados nos permiten corroborar una tesis que ya hemos señalado en otros trabajos, en términos de que concebimos a las remesas como una variable macroeconómica anticíclica e inflexible a la baja (Canales y Montiel, 2004). El carácter anticíclico además, nos permite reforzar nuestra tesis general de entenderlas como un ingreso familiar, más que como un fondo de inversión.

- Por último, resulta interesante comprobar también que, sistemáticamente, las remesas no muestran ninguna relación estadísticamente significativa con las variables financieras y de inversión. En efecto, en todos los casos, éstas no parecen estar determinadas por el comportamiento de la tasa de interés, tanto en el país de origen como en el de destino de la migración, así como tampoco suelen tener un comportamiento similar al de otros fondos de inversión, como lo es la Inversión Extranjera Directa.

En otras palabras, el modelo econométrico nos indica que no hay evidencia estadística que permita definir a las remesas como un fondo de inversión productiva. Por el contrario, nos indica que el comportamiento macroeconómico de las mismas no guarda ningún tipo de relación estadísticamente significativa con los determinantes tradicionales de la inversión productiva.

En síntesis, el análisis de los determinantes de las remesas nos permite concluir que en términos macroeconómicos, su dinámica y comportamiento no corresponden al de un fondo de ahorro o inversión; sino más bien al de un ingreso familiar que, bajo la forma de transferencias familiares, contribuye a compensar los efectos negativos de las devaluaciones recurrentes de la moneda, la pérdida de poder adquisitivo de los salarios, y la pérdida de competitividad de nuestras eco- 
nomías, provocadas por las crisis reiteradas y los ciclos recesivos de las economías latinoamericanas.

\section{CONCLUSIONES}

Las remesas son, que duda cabe, una fuente importante de ingreso para las familias perceptoras. Si a ello agregamos la magnitud que han alcanzado en los últimos años, no debe extrañarnos, entonces, el optimismo que se trasluce en el discurso de gobiernos nacionales y organismos internacionales. Es común leer informes de organismos públicos, y escuchar declaraciones de funcionarios de gobierno, en donde se señala el significativo aporte de las remesas en la reducción de la pobreza, promoción del desarrollo y el bienestar de las familias, entre muchos otros supuestos beneficios (SEGIB, 2006; Banco Mundial, 2004; CEPAL, 2000).

Sin embargo, cuando se revisan con más detalle, incluso los mismos informes de dichas instituciones, se observa en cambio, que este optimismo se sustenta más en un conjunto de buenos deseos y mejores intenciones, que en datos estadísticos y evidencia empírica. En este sentido, en este trabajo hemos querido documentar con datos sobre la experiencia latinoamericana, una visión crítica, que no pesimista, sobre el papel e impacto de las remesas.

$\mathrm{Al}$ respecto, un primer punto que resalta es que no parecen existir ni argumentos lógicos (teóricos) ni empíricos que permitan inferir que las remesas pudieran tener un papel relevante como promotoras y/o financiadoras del desarrollo económico. Por el contrario, como hemos mostrado en este texto, el comportamiento macroeconómico de éstas es mucho más próximo al de un tipo de transferencias salariales que a un fondo de inversión familiar o privado. Asimismo, estas transferencias salariales suelen ser mayores y más importantes en aquellos países con mayores niveles de pobreza y menor nivel de desarrollo. Por lo mismo, la inversión productiva financiada con remesas no sólo no llega a los montos necesarios para impulsar un proceso de desarrollo, sino que además se da en ambientes macroeconómicos muy desfavorables que limitan y restringen sus impactos reales.

Si consideramos que la migración es un fenómeno esencialmente laboral, entonces es lógico suponer que las remesas constituyen el mecanismo a través del cual los salarios de los trabajadores migrantes son transferidos hacia sus hogares en los países de origen. Por lo mismo, lo más lógico es esperar que estas transferencias salariales sean usadas para sustentar el consumo familiar. Asimismo, no hay razón lógica ni empírica que permita suponer que estas familias han de tener un comportamiento y racionalidad económica diferentes a los de otras familias no perceptoras de remesas. En otras palabras, para ellas las remesas configuran un fondo salarial, que como en la gran mayoría de las familias de trabajadores, constituyen la base de sus ingresos familiares. De esta forma, estas transferencias sa- 
lariales podrán contribuir a mejorar el nivel de vida de los hogares perceptores, pero están muy lejos de representar una estrategia que permita superar y resolver los problemas estructurales que perpetúan la pobreza (Canales, 2007a; Székely y Rascón, 2004).

Ahora bien, a estas limitaciones de las remesas se suma otro factor de no poca importancia. Las remesas, en el fondo, reflejan además un marcado carácter de clase. No sólo son un fondo salarial, sino que corresponden a ingresos salariales de trabajadores que combinan una inserción laboral de alta vulnerabilidad y precariedad en Estados Unidos, con una condición de pobreza, marginación y vulnerabilidad social en sus países de origen.

En síntesis, el bajo monto mensual por transferencia que percibe cada familia nos permite entender el carácter y significado económico y social de las remesas. Por un lado, son un ingreso salarial, que como cualquier otro, se destina al consumo familiar. Por otro lado, el reducido monto promedio por hogar perceptor nos indica, además, que se trata principalmente de familias y trabajadores de bajos recursos, inmersos en situaciones de vulnerabilidad social y precariedad económica. Son estratos pobres, con muchas carencias, y en donde las remesas pueden contribuir a paliar esta situación de pobreza, pero en ningún caso a resolverla.

\section{BIBLIOGRAFÍA}

Acosta, Pablo; César Calderón, Pablo Fajnzylber y Humberto López (2006), «Remittances and Development in Latin America», The World Economy, 29(7): 957-987.

ADAms H., Richard Jr. y J. Page (2005), «Do international migration and remittances reduce poverty in developing countries?», World Development, 33(10): 1645-1669.

Adelman, Irma y Edward Taylor J. (1990), «Is Structural Adjustment with a Human Face Possible? The Case of Mexico", Journal of Development Studies 26(3): 387-407.

Alleyne, Dillon, Claremont D. Kirton y Mark Figueroa (2008), «Macroeconomic Determinants of Migrant Remittances to Caribbean Countries: Panel Unit Roots and Cointegration», JEL, Classifications: D,E60,C23. 137-153. Retrieved from ProQuest database.

Banco Mundial (2004), La pobreza en México: una evaluación de las condiciones, las tendencias y la estrategia del Gobierno, México, Banco Mundial.

BECK, Ulrich (2000), Un nuevo mundo feliz. La precariedad del trabajo en la era de la globalización, Barcelona, Paidós.

Binford, Leigh (2002), «Remesas y subdesarrollo en México», Relaciones. Estudios de Historia y Sociedad, 23(90): 117-158.

Canales, Alejandro I. (2007a), «Migrant Remittances; Savings Funds or Wage Income». en G. Zárate-Hoyos, Multidisciplinary Perspectives on Remittances form Migrant Workers in the United States, Alemania, Kassel University Press, pp. 59-98.

(2007b), «Envois de fonds des migrants, développement et pauvreté. Une vision critique à partir de l'Amérique latine», en I. Yepes y G. Herrera, Nouvelles migrations latinoamericaines en Europe, OBREAL, Universidad Católica de Lovaina. 
e Israel Montiel Armas (2004), «Remesas e inversión productiva en comunidades de alta migración a Estados Unidos. El caso de Teocaltiche, Jalisco», Migraciones internacionales, 2(3): 142-172.

CARLING, Jorgen (2007), «Interrogating remittances: Core questions for beeper insight and better policies», Migration and Development: Perspectives from the South, Conference Proceedings, Bellagio 2006-2007, Study and Conference Center.

CAstells, Manuel (1998), "La era de la información», Economía, sociedad y cultura, vol. 1, La sociedad red, España, Alianza Editorial.

Castles, Stephen y Mark J. Miller (1993), The Age of migration. International Population Movements in the Modern World, Nueva York, Guilford Press.

CEPAL (2006), Migración internacional, derechos humanos y desarrollo, LC/W.98, Santiago, Comisión Económica para América Latina y El Caribe.

(2005), Panorama social de América Latina, Santiago, Comisión Económica para América Latina y El Caribe.

(2000), Uso productivo de las remesas familiares y comunitarias en Centroamérica (LC/MEX/L.420), México.

ChAmI, Ralph, Connel Fullenkamp y Samir Jahjah (2003), Are Immigrant Remittances Flows a Source of Capital for Development?, IMF Working Paper WP/03/189, Washington, Fondo Monetario Internacional.

Cortina, Jerónimo, Rodolfo de la Garza y Enrique Ochoa-Reza (2004), «Remesas: límites al optimismo", Foreign Affairs en Español, 5(3): 27-36.

De HaAs, Hein (2007), Remittances, Migration and Social Development. A Conceptual Review of the Literature, United Nations, Research Institute for Social Development. Social Policy and Development Programme Paper Number 34.

Delgado Wise, Raúl, Humberto Márquez Covarrubias y Héctor Rodríguez Ramírez (2004), "Organizaciones transnacionales de migrantes y desarrollo regional en Zacatecas», Migraciones Internacionales, 7: 159-181.

FMI (2005), World Economic Outlook, April 2005, International Monetary Fund, Washington.

FOMIN (2004), Remittances to Latin America and the Caribbean: Goals and Recommendations, Washington, Fondo Multilateral de Inversiones, Banco Interamericano de Desarrollo.

GARCía Zamora, Rodolfo (2005), "Migración internacional y remesas colectivas en Zacatecas», Foreign Affairs en Español, 5 (3).

GoldRing, Luin (2005), «Implicaciones sociales y políticas de las remesas familiares y políticas», en Raúl Delgado Wise y Beatrice Knerr (coordinadores), Contribuciones al análisis de la migración internacional y el desarrollo regional en México, México, Universidad Autónoma de Zacatecas y Miguel Ángel Porrúa.

Hinojosa Ojeda, Raúl (2003), «Transnational Migration, Remittances and Development in North America: Globalization Lessons from the OaxaCalifornia Transnational Village/Community Modeling Project», Paper prepared for the Conference Remittances as a Development Tool in Mexico; Multilateral Investment Fund; Inter-American Development Bank, Mexico City.

Hugo, Graeme (2005), «Experiencias asiáticas con las remesas», en Donald F. Ferry y Steven R. Wilson (editores), Remesas de inmigrantes. Moneda de cambio económico y social, Washington, Banco Interamericano de Desarrollo. 
Jones, Richard C. (1998), «Remittances and Inequality: A Question of Migration Stage and Geographic Scale», Economic Geography, 74(1): 8-25.

Lozano, Fernando (2005), «De excluidos sociales a héroes sexenales. Discurso oficial y remesas en México», en Raúl Delgado Wise y Beatrice Knerr (coordinadores), Contribuciones al análisis de la migración internacional y el desarrollo regional en México, México, Universidad Autónoma de Zacatecas y Miguel Ángel Porrúa.

Martínez Pizarro, Jorge (2003), «Panorama regional de las remesas durante los años noventa y sus impactos macrosociales en América Latina», Migraciones Internacionales, núm. 5: 40-76.

Moser, Caroline O.N. (1998), «The asset vulnerability framework: Reassessing urban poverty reduction strategies", World Development, 26(1): 1-20.

NAïr, Sami (2006), Y vendrán... Las migraciones en tiempos hostiles, Barcelona, Planeta.

Orozco, Manuel (2004), Remittances to Latin America and the Caribbean: Issues and perspectives on development, Report Commissioned by the Organization of American States, septiembre, Washington, Organization of American States.

y Steven R. Wilson (2005), «Para que las remesas produzcan resultados», en Donald

F. Ferry y Steven R. Wilson (editores), Remesas de inmigrantes. Moneda de cambio económico y social, Washington, Banco Interamericano de Desarrollo.

Piore, Michael (1979), Birds of Passage, Cambridge, Cambridge University Press.

RatHA, Dilip (2003), «Worker's Remittances: An Important and Stable Source of External

Development Finance», Global Development Finance 2003, Washington, World Bank.

Reinke, Jans y Neil Patterson (2005), «Remittances in the Balance of Payments Framework», documento del International Technical Meeting on Measuring Remittances, World Bank, Washington, 24-25 de enero de 2005.

Sassen, Saskia (1998), Globalization and its Discontents, Nueva York, The New Press.

SEGIB (2006), Unidos por las Migraciones, Madrid, Secretaría General Iberoamericana.

SzéKelY, Miguel y Erica Rascón (2004), «México 2000-2002: reducción de la pobreza con estabilidad y expansión de programas sociales», Serie Documentos de Investigación núm. 15, México, Secretaría de Desarrollo Social.

TAYLOR, J. Edward y T.J. Wyatt (1996) «The shadow value of migrant remittances, income and inequality in a household-farm economy», Journal of Development Studies, vol. 32, núm. 6, pp. 899-912.

Terry, Donald (2006), «Las Remesas como Instrumento de Desarrollo», Unidos por las migraciones, Madrid, Secretaría General Iberoamericana.

Torres, Federico (2001), «Uso productivo de las remesas en México, Centroamérica y República Dominicana, Experiencias recientes», La migración internacional y el desarro$1 l o$ en las Américas, Santiago, Chile, CEPAL, BID, OIM, FNUAP.

Zárate-Hoyos, Germán (2007), "A Mutiplier Analysis of Remittances in the Mexican Economy», en G. Zárate-Hoyos (editor), Multidisciplinary Perspectives on Remittances form Migrant Workers in the United States, Alemania, Kassel University Press, pp. 102-129.

Zlolniski, Christian (2006), Janitors, Street Vendors, and Activists: The Lives of Mexican immigrants in Silicon Valley, Berkeley, University of California Press. 